PEB Échanges, Programme pour la construction et l'équipement de l'éducation 2002/02

\title{
Le City and Islington College, au Royaume-Uni
}

\section{Grace Kenny}




\section{LE CITY AND}

\section{ISLINGTON COLLEGE, AU ROYAUME-UNI}

\section{Le cadre d'action}

Le gouvernement britannique reconnaît l'extrême diversité des activités de formation s'offrant aux groupes de population n'ayant pas fait d'études universitaires et ayant dépassé l'âge de l'enseignement obligatoire ; sous I'influence de rapports tels que celui de Claus Moser ${ }^{1}$ sur les compétences de base des adultes et celui de Helena Kennedy ${ }^{2}$ sur l'élargissement de la participation aux activités de formation tout au long de la vie, le gouvernement a décidé de regrouper la pléthore de dispositifs existants en une politique cohérente visant à améliorer le niveau de formation et les qualifications à tous les âges. Comme le précise le nouveau Learning and Skills Council dans son plan d'action, " A l'heure actuelle, notre position est très inférieure à la moyenne des pays de l'OCDE par rapport à certains indicateurs clés tels que la participation des jeunes à des activités de formation ${ }^{3}$.»

Le message transmis aux établissements existants, bien établis, de formation permanente est qu'ils doivent réagir aux « initiatives » des pouvoirs publics en évolution constante tout en s'efforçant de préserver et d'améliorer leurs propres atouts et efficacité. De tous les établissements d'enseignement, depuis les écoles maternelles jusqu'aux universités, les établissements de formation permanente sont ceux qui souffrent le plus (et de temps à autre qui bénéficient le plus) des antagonismes existant entre les priorités nationales et l'exigence d'adaptation au niveau local.

Le City and Islington College illustre tous ces conflits potentiels et possibilités d'adaptation.

Le City and Islington College, situé au cœur de Londres, a été constitué en 1993 par la fusion de divers établissements locaux parmi lesquels un « sixth form college » (établissement proposant un enseignement théorique général aboutissant à la délivrance de diplômes d'État et permettant d'accéder à l'université), deux établissements de formation générale postscolaire et un centre de formation pour adultes. Le College qui assure des formations à quelque 4700 étudiants à plein temps et à 16500 autres à temps partiel est actuellement réparti sur plus de dix sites.

Non seulement les enseignants passent beaucoup de temps à se déplacer d'un bout à l'autre de l'un des centres urbains les plus encombrés d'Europe, mais en outre les élèves pâtissent d'avoir à utiliser un ensemble de bâtiments éparpillés, construits entre les années 1880 et 1970, dont certains n'ont même pas été conçus pour l'enseignement. Quatre de ces bâtiments sont de beaux exemples d'écoles à plusieurs étages construites par le London School Board en application de la loi de 1870 sur l'enseignement élémentaire. Toutefois, aussi magnifiques soient-ils, ces bâtiments avaient été conçus pour des enfants et non pas des adolescents et des adultes et ne disposent donc pas des infrastructures voulues; ils ne disposent pas en particulier d'installations de technologies de l'information (TI) pour l'enseignement et l'acquisition de savoirs, ni d'ailleurs de lieux de vie sociale. L'âge et l'état de ces bâtiments sont à l'origine de la seule note faible attribuée au College lors de sa dernière inspection.

Le College s'est donc lancé dans un vaste programme $d^{\prime}$ investissements financiers, qui répondra aux toutes dernières exigences du gouvernement tout en tirant parti des atouts particuliers de l'établissement en matière de formation.

\section{Le programme}

\section{Rationalisation}

En premier lieu, le College a concentré ses formations sur trois principaux sites bien desservis par les transports en commun : les sites de Angel dans le sud, de Finsbury Park dans le nord et de Holloway dans le centre. Grâce à cette concentration, la superficie intérieure brute du College passera de 38000 à $35000 \mathrm{~m}^{2}$.

Ce programme qui prévoit à la fois des constructions nouvelles et des remises à neuf aura un coût de l'ordre de 50 millions GBP, dont $80 \%$ pourront bénéficier d'une aide partielle de l'État (à concurrence de 35 \%).

\section{Planification}

Pour obtenir le chiffre de $35000 \mathrm{~m}^{2}$, on a utilisé la méthode de planification du Further Education Funding Council ${ }^{4}$. Auparavant, la superficie était calculée en fonction de l'effectif d'élèves ou du nombre d'élèves, converti en surface équivalente plein temps. Toutefois, dans la mesure où les modes de fréquentation se sont de plus en plus diversifiés, le Council recommande de convertir le nombre annuel total $d$ 'heures de formation encadrées en un nombre minimum de places. Ce nombre est « virtuel » car il représente le nombre de places qui serait nécessaire si toutes les places pouvaient être utilisées pendant la tota-

1. Department for Education and Employment (1999), « Improving Literacy and Numeracy - A Fresh Start », Londres.

2. Further Education Funding Council (1999), "Learning Works», Coventry.

3. Learning and Skills Council (2001), "Strategic Framework to 2004, Corporate Plan », juillet, Coventry.

4. Further Education Funding Council (1997), Supplement to Circular 97/37, « Guidance on Floorspace Management in Further Education Colleges », novembre, Coventry. 
lité (100\%) de l'année d'enseignement (1 440 heures actuellement). Ce chiffre est corrigé pour tenir compte du taux d'utilisation que l'on peut raisonnablement escompter (40\% par exemple), ce qui indique le nombre total de places à prévoir. Le nombre ainsi calculé est alors réparti en types d'espace et se voit appliquer une norme d'espace par place ${ }^{5}$; il est alors possible de calculer l'espace qui n'est destiné ni à l'enseignement ni à l'apprentissage, celui qui est réservé à la vie de proximité ainsi que celui que nécessite un aménagement équilibré ${ }^{\text {. }}$

Un type d'espace qui revêt de l'importance pour le secteur de la formation permanente est à présent le centre de ressources documentaires où les jeunes adultes peuvent étudier en dehors de la structure rigide de la salle de classe tout en étant supervisés. Le Council souhaiterait que les espaces "formation » (bibliothèque et centre de ressources documentaires) représentent entre 10 et $20 \%$ de la superficie intérieure brute totale de tout établissement d'enseignement. Il suggère également de prévoir pour le nombre minimum de places diverses aires qui devraient permettre de mener les activités d'enseignement et d'acquisition de savoirs propres aux établissements " normaux » d'enseignement postscolaire (11 à 14.5 m², le chiffre inférieur étant privilégié), et tout le problème est d'harmoniser ces normes indicatives avec les calculs établis à partir des différentes formations et des exigences spécifiques.

\section{Principes généraux d'aménagement}

Dans le cas du City and Islington College, afin de faire correspondre les orientations globales et les exigences spécifiques et de favoriser l'adaptabilité et la flexibilité dont il est sans cesse question, certains principes ont été adoptés :

1. Conserver une forte proportion d'espaces pour les activités d'enseignement proprement dites.

2. Ne pas affecter des espaces d'enseignement général à des disciplines spécifiques.

3. Situer le centre de ressources documentaires à proximité du centre de documentation et d'information (CDI) et des salles informatiques pour que le CDI puisse s'élargir ou que le centre de ressources documentaires puisse être converti à d'autres usages.

4. Éviter d'implanter des espaces destinés à des activités spécialisées sur plus d'un site et s'assurer que ces espaces peuvent être réaffectés à d'autres usages.

5. Department of Education and Science (1983), Design Note 33, "Area Guidelines for Sixth Form, Tertiary and NAFE Colleges », Londres.

6. Department of Education (1994) Design Note 50, «Accommodation for Changes in Further Education », Londres.
5. Éviter de construire des murs porteurs à l'intérieur pour qu'il soit possible si nécessaire d'agrandir ou de rétrécir les diverses aires.

6. Recourir à des systèmes de programmation pour optimiser I'utilisation de l'espace dans le temps.

\section{Les projets}

Le site Angel (Superficie intérieure brute : $11500 \mathrm{~m}^{2}$ )

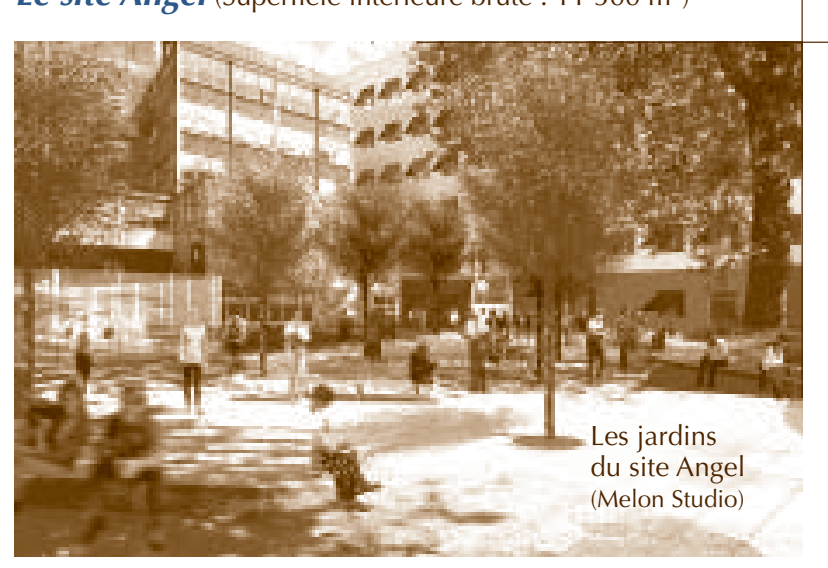

\section{Centre phare d'études secondaires terminales}

Ce centre est une construction entièrement nouvelle comprenant six étages et une façade de 90 mètres de longueur donnant sur une voie à grande circulation ; les parois extérieures sont construites en Kalwall, matériau translucide, et comprennent des parties vitrées transparentes. Une partie du bâtiment donnant sur la rue sera occupée par un commerce de proximité de petite taille. Les jardins existants seront préservés et le cas échéant réaménagés pour fournir une diversité d'espaces ouverts, sécurisés et accessibles au public.

Le Centre accueillera des élèves préparant des niveaux " $A$ » et « AS » (16-19 ans) durant la journée et des élèves adultes dans la soirée. Les filières proposées aux élèves âgés de 16 à 19 ans sont diverses comprenant aussi bien les langues modernes que la musique et les beaux-arts. Même si actuellement l'enseignement est pour l'essentiel dispensé dans une salle fermée de type traditionnel, les aires seront aménagées de façon à pouvoir s'adapter si besoin est à une formation de type plus documentaire. Les élèves adultes utiliseront les salles de classe classiques et quelques zones réservées à des disciplines spécialisées dans la

Façade du nouveau site Angel (van Heyningen et Haward) 
soirée. En outre, le théâtre (situé dans le bâtiment adjacent déjà existant) servira pour donner des spectacles accessibles au public.

Les architectes sont van Heyningen et Haward. Le permis de construire a été obtenu et les travaux commenceront en février 2002.

\section{Centre de qualification et de perfectionnement professionnels}

Un bâtiment datant du début des années 60, occupé tout d'abord par un établissement d'enseignement secondaire puis utilisé à une date plus récente par la City University, sera remis à neuf pour accueillir les laboratoires et ateliers spécialisés requis pour les formations aux métiers de l'optique et d'autres activités de perfectionnement professionnel (parmi lesquelles la formation à l'utilisation des TI et à l'action commerciale, ainsi que l'apprentissage de l'anglais pour les personnes de langue étrangère et l'acquisition de compétences de base pour la population locale).

Les architectes sont Gollifer Associates.
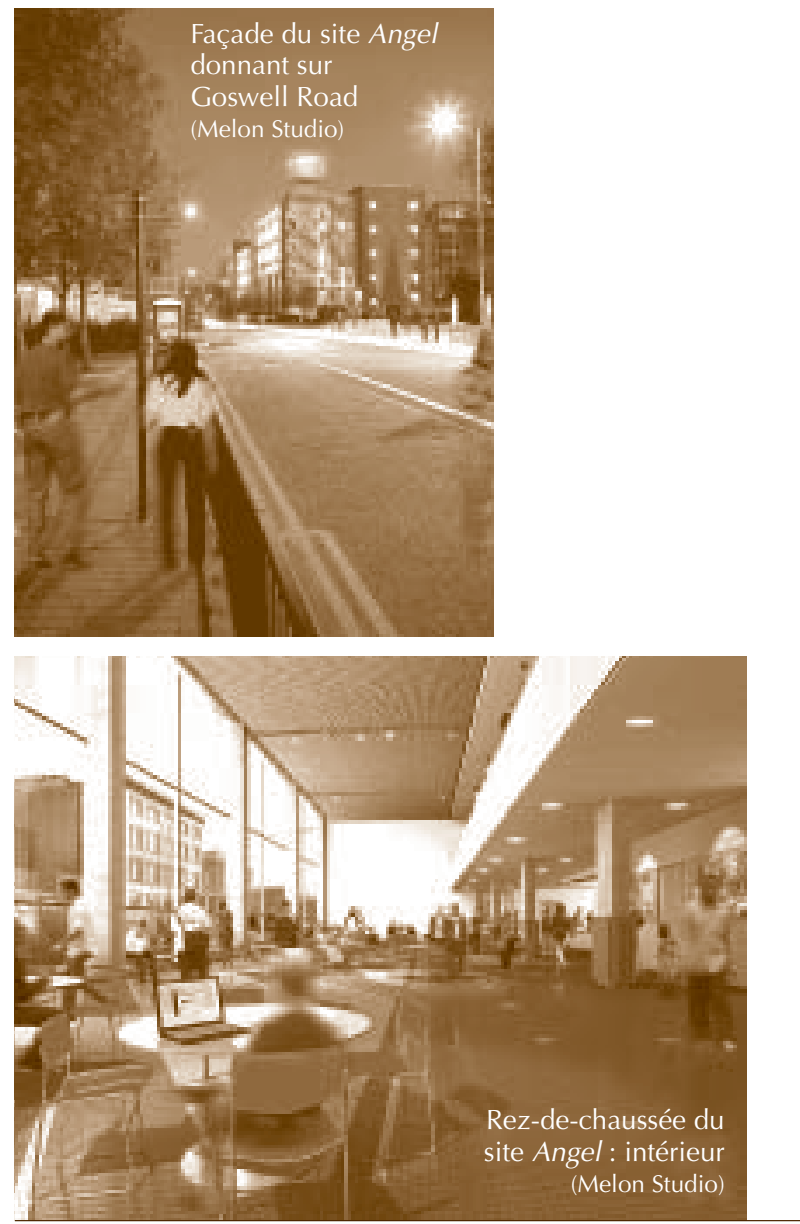

Finsbury Park (Superficie intérieure brute : $6900 \mathrm{~m}^{2}$ )

\section{Centre de formation permanente}

Ce site sera le premier grand nouveau centre de formation pour adultes construit dans le nord de Londres depuis une génération. Il proposera des formations complètes dans des domaines très variés parmi lesquels les technologies de l'information, les compétences de base et I'anglais (pour les demandeurs $d^{\prime}$ asile entre autres) mais aussi la remise en forme et les soins de beauté, la céramique et la fabrication de meubles. Ce site accueillera également le « projet restauration ». II s'agit d'une formation à temps partiel conçue pour les adultes qui ont besoin d'acquérir des compétences de base utiles pour la vie de tous les jours : des petits groupes viennent deux ou trois jours par semaine, font les courses, préparent les repas et les servent aux autres utilisateurs du site.

La seule école victorienne que compte encore le College se trouve sur ce site et sera préservée dans le nouveau plan d'aménagement qui prévoit de vider partiellement l'intérieur du bâtiment et de l'encadrer d'une nouvelle construction. L'atrium ainsi obtenu sera occupé par une cafétéria, un cyber-café et quelques équipements de $\mathrm{TI}$ en libre-service ; il accueillera également un espace repas qui sera de temps à autre utilisé par le projet « restauration ».

Les départements utilisant des équipements lourds seront installés au rez-de-chaussée alors que les locaux réservés au centre de remise en forme et de soins de beauté seront installés aux étages supérieurs dans un appartement autonome, pour préserver l'intimité, composé de grandes pièces. Les salles nécessitant un dispositif de climatisation (principalement celles qui comprennent les équipements de TI) se trouveront dans les nouvelles zones fermées, donnant sur Blackstock Road, rue très commerçante.
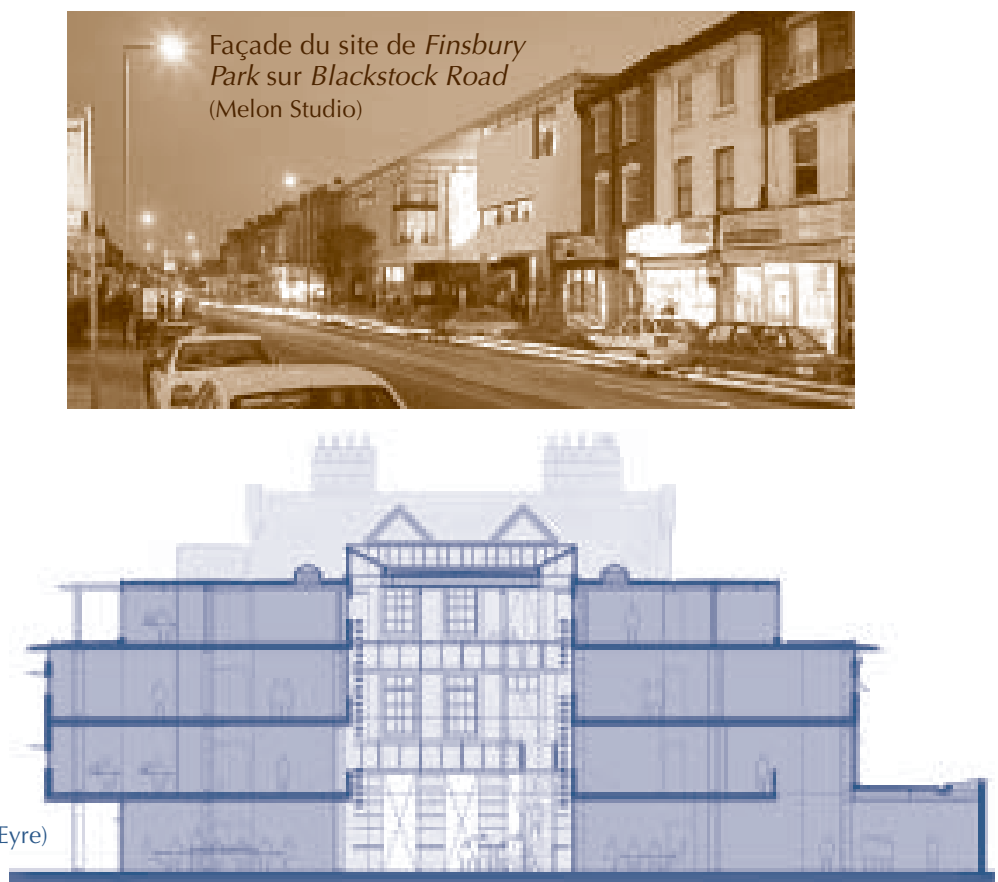
Ce projet se trouve au stade de l'étude de faisabilité et les architectes sont Wilkinson Eyre.

\section{A propos de l'ensemble des sites}

Sur chacun des sites, une attention particulière est accordée, au stade de la conception, aux équipements sociaux et aux installations d'accompagnement dont ont besoin le personnel et les étudiants. Holloway et Finsbury Park disposent chacun d'une crèche; les salles réservées au personnel sont conçues pour seulement huit à dix utilisateurs ; les aires réservées à la vie sociale, animée ou tranquille, dans le College correspondent à l'âge et aux caractéristiques des différents utilisateurs ; les dispositifs de sécurité sont discrets. Ayant commandé des bâtiments à des architectes dont les styles sont très différents, le College s'informe sur la façon de présenter une image de marque (dans laquelle les couleurs pourraient être un indicateur important) de telle sorte que les passants voient d'emblée de quel établissement il s'agit.

mun certaines ressources et on espère que les utilisateurs de la bibliothèque municipale seront tentés d'aller suivre une formation au College.

Les architectes sont Wilkinson Eyre. Le permis de construire a été accordé en septembre 2001 et les travaux devraient commencer en 2002.

\section{Enseignements et conclusions}

- Comprendre que la théorie (les lignes directrices, les normes en matière d'espace, les objectifs visés, etc.) ne suffit pas. La théorie donne un point de départ utile mais doit être tempérée par les applications pratiques et le pragmatisme.

- Reconnaître que le budget doit couvrir beaucoup plus que les frais de construction; prévoir au moins $40 \%$ de fonds supplémentaires pour l'acquittement des honoraires, les aménagements, les déménagements, les frais obligatoires et les honoraires de conseillers, et les imprévus.

- Former l'équipe souhaitée, peu à peu si nécessaire.

- Maintenir chaque personne dans le circuit.

- Répertorier tous les risques ; le faire avec honnêteté et prévoir le pire.

- Veiller à ne pas surestimer l'espace requis. En réalité, l'espace est toujours moins utilisé qu'on ne le pense.

Sites Web :

City and Islington College, www.candi.ac.uk Learning and Skills Council, www.lsc.gov.uk

Departmentfor Education and Skills, www.teachernet.gov.un/ schoolbuildings

Cet article a été rédigé par Grace Kenny qui sera heureuse de répondre à vos questions et de connaître vos points de vue.

Grace Kenny

Londres, Royaume-Uni

Tél./télécopie : 442073859132

gracekenny@dial.pipex.com

ou pmarsh@candi.ac.uk 